\title{
ANTI-WINDUP SCHEME FOR PRACTICAL CONTROL OF POSITIONING SYSTEMS
}

\author{
WAHYUdi*, TARig FAISAl AND AbDUlgani Albagul \\ Department of Mechatronics Engineering, International Islamic University, Malaysia, Jalan \\ Gombak, 53100 Kuala Lumpur, Malaysia \\ e-mail:wahyudi@iiu.edu.my,tarig_2@hotmail.com,albagul@iiu.edu.my
}

\begin{abstract}
Positioning systems generally need a good controller to achieve high accuracy, fast response and robustness. In addition, ease of controller design and simplicity of controller structure are very important for practical application. For satisfying these requirements, nominal characteristic trajectory following controller (NCTF) has been proposed as a practical point-to-point (PTP) positioning control. However, the effect of actuator saturation can not be completely compensated for due to the integrator windup as the plant parameters vary. This paper presents a method to improve the NCTF controller for overcoming the problem of integrator windup using simple and classical tracking anti-windup scheme. The improved NCTF controller is evaluated through simulation using a rotary positioning system. The results show that the improved NCTF controller is adequate to compensate for the effect of integrator windup.
\end{abstract}

Keywords: Positioning, point-to-point, integrator windup, compensation, controller, robustness.

\section{Introduction}

Motion control systems play important roles in industrial process such as machine tools, semiconductor manufacturing systems and robotic systems. One type of motion control systems is the point-to-point (PTP) positioning system, which is used to move a plant from one point to another. The positioning systems generally need a good controller to satisfy some stringent requirements such as high accuracy, fast response and robustness. Up to now many types of controllers have been proposed and evaluated for positioning systems; for example the model following type controller such as controllers with disturbance observer [1-4], time-optimal controllers [5-8] and sliding mode controllers $[9,10]$. These controllers would give good positioning performance in cases an expert in motion control system designs the controller using the exact model and value of its parameters. In general, advanced controllers tend to be complicated and require deep knowledge of controller theory. However, in practical applications, engineers who are not expert in control system often need to design controller. In addition, exact modeling and parameter identifications are generally troublesome and time consuming tasks. Hence,

\footnotetext{
* Correspondence author
} 
ease of controller design and simplicity of controller structure are very important in practical application.

In order to overcome these problems, nominal characteristic trajectory following (NCTF) controller has been proposed as a practical controller for point-to-point (PTP) positioning systems [11]. It has been shown that, the NCTF control system has a good positioning performance and robustness $[12,13]$. The NCTF controller is also effective in compensating the effect of the friction which is a source of positioning inaccuracy [14]. However, the effect of actuator saturation can not be completely compensated for due to integrator windup as a result of the variation of the plant parameters [15]. The NCTF controller gives an excessive overshoot when both actuator saturation and parameter variations (especially inertia variation) occur in the positioning systems.

This paper describes a method to improve the NCTF controller for overcoming the degradation of the positioning performance due to the integrator windup. First, NCTF control concept and its controller design procedure are introduced. Then, an improved compensator for overcoming the integrator windup is described. Finally, the effectiveness of the improved NCTF controller is examined by simulation.

\section{NCTF control concepts}

The structure of the NCTF control system is shown in Fig. 1. The NCTF controller consists of a nominal characteristic trajectory (NCT) and a compensator. The NCTF controller works under the following two assumptions:

A DC or an AC servomotor is used as an actuator of the plant.

PTP positioning systems are chosen, so $\theta_{r}$ is constant and $\dot{\theta}_{r} \equiv 0$.

Here, the objective of the NCTF controller is to make the plant motion follows the NCT and end at the origin of the phase plane $(e, \dot{e})$.

Figure 2 shows an example of a plant motion controlled by the NCTF controller. The motion comprises two phases. The first one is the reaching phase whilst the other one is the following phase. In the reaching phase, the compensator forces the plant motion to reach the NCT as fast as possible. However, in the following phase the compensator controls the plant motion so as to follow the NCT and end at the origin. The plant motion stops at the origin, which represents the end of the positioning motion. Thus, in the NCTF control system, the NCT governs the positioning response performance.

The NCTF controller is designed based on a simple open-loop experiment of the plant as follows:

Open-loop-drive the plant with stepwise inputs and measure the displacement and velocity responses of the plant. 


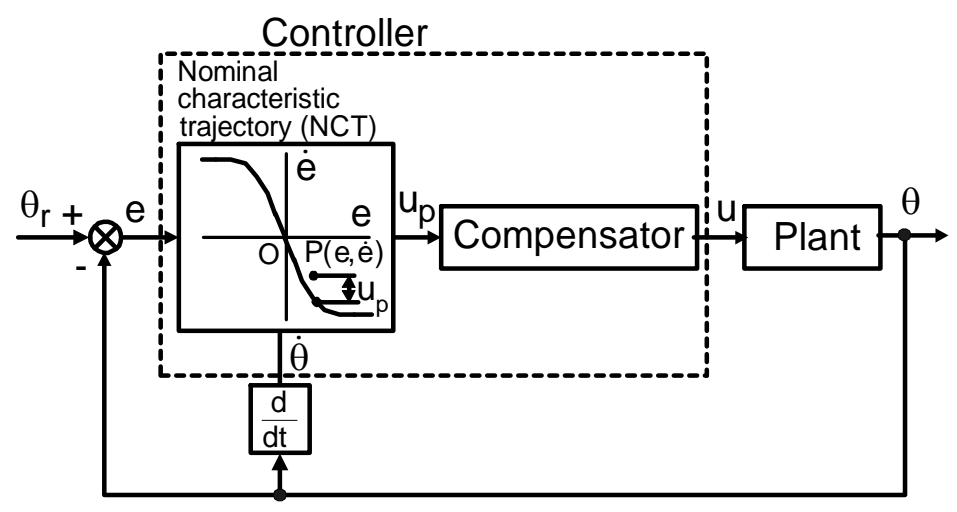

Fig. 1: NCTF control system.

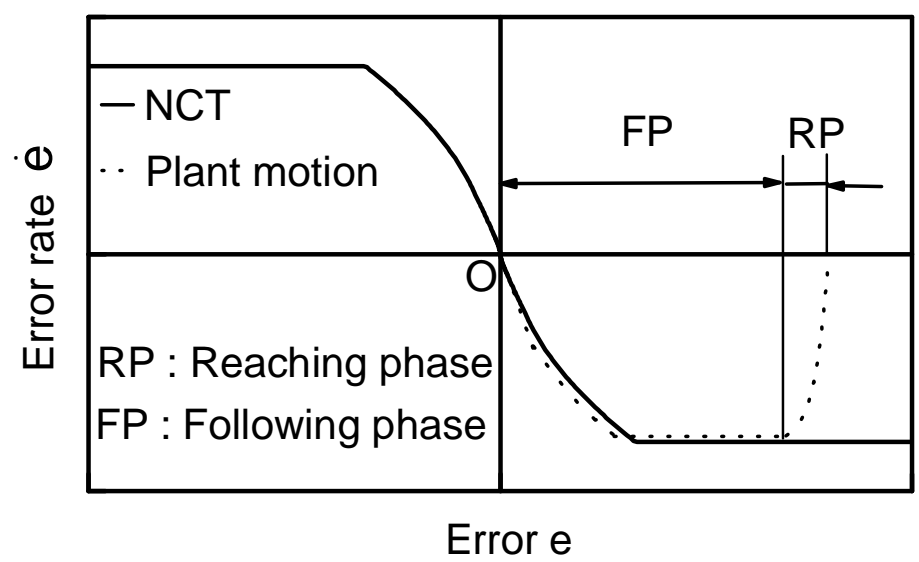

Fig. 2: NCT and plant motion.

Figure 3(a) shows the stepwise inputs, and the velocity and displacement responses due to the stepwise inputs. In this paper, the rated input to the actuator $u_{r}$ is used as the height of the stepwise inputs.

Construct the NCT by using the plant responses.

The velocity and displacement responses are used to determine the NCT. Since the main objective of PTP system is to stop the plant at a certain position, a deceleration process is used, see curve A, Fig. 3(a). The h in Fig. 3 represents the maximum velocity. From the curve in the area A and $\mathrm{h}$ in Fig. 3(a), the NCT in Fig. 3(b) is determined. Since the NCT is constructed based on the actual responses of the plant, the NCT includes nonlinearity effects such as friction and saturation. The important NCT information, which will be used to design the compensator, are NCT inclination $\mathrm{m}$ near the origin and maximum error rate of $\mathrm{h}$. In this case, from the relationship between plant dynamics of Eq. (1) and Fig. 3(b), it is clear that the inclination near origin $m$ and the maximum error rate $h$ relate with parameters of the plant as follows $[12,15]$ : 


$$
\begin{gathered}
K=-\frac{h}{u_{r}} \\
\alpha=-m
\end{gathered}
$$

Design the compensator based on the NCT information.

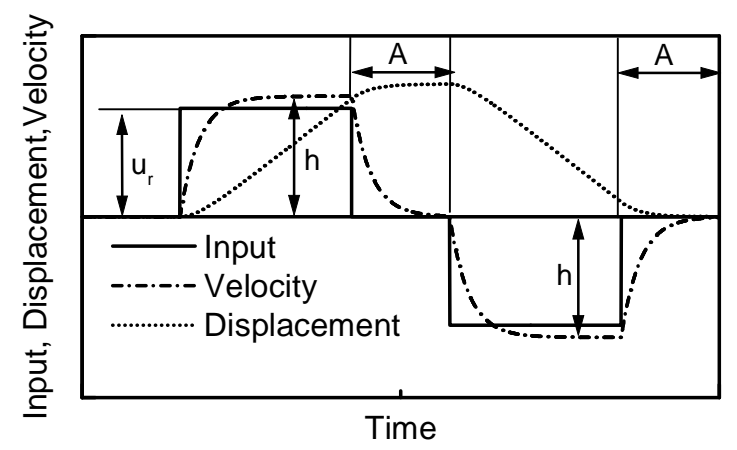

(a) Stepwise inputs and responses

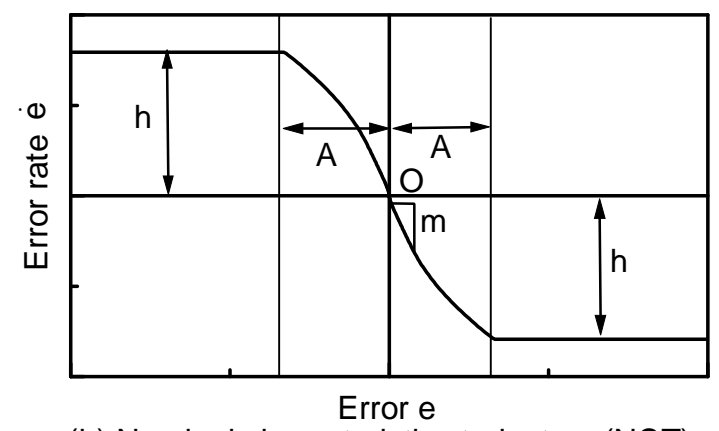

(b) Nominal characteristics trajectory (NCT)

Fig. 3: NCT determination.

Here, the following PI compensator is adopted due to its simplicity:

$$
u=K_{p} u_{p}+K_{i} \int u_{p} d t
$$

where $\mathrm{K}_{\mathrm{p}}$ and $\mathrm{K}_{\mathrm{i}}$ are proportional and integral gains respectively. Using the PI compensator parameters $\mathrm{K}_{\mathrm{p}}$ and $\mathrm{K}_{\mathrm{i}}$, and the NCT characteristic near the origin (see Fig. 3(b)), the transfer function of the closed-loop positioning system controlled by the NCTF controller can be approximated as follows [11-15]:

$$
\frac{\Theta(s)}{\Theta_{r}(s)}=G(s)=G_{1}(s) G_{2}(s)
$$

where 


$$
\begin{aligned}
& G_{1}(s)=\frac{\alpha}{s+\alpha} \\
& G_{2}(s)=\frac{2 \zeta \omega_{n}+\omega_{n}^{2}}{s^{2}+2 \zeta \omega_{n}+\omega_{n}^{2}} \\
& K_{p}=\frac{2 \zeta \omega_{n}}{K \alpha} \\
& K_{i}=\frac{\omega_{n}^{2}}{K \alpha}
\end{aligned}
$$

When $\zeta$ and $\omega_{\mathrm{n}}$ are large enough, $\mathrm{G}(\mathrm{s})$ becomes nearly equal to $\mathrm{G}_{1}(\mathrm{~s})$, which represent the condition when the plant motion follows the NCT as the objective of the NCTF control system. Moreover, large $\zeta$ and $\omega_{\mathrm{n}}$ also make the closed-loop system robust to friction or inertia variation of the plant in continuous systems [9]. Finally, by using $\zeta$ and $\omega_{\mathrm{n}}$ as design parameters and considering Eqs. (2) and (3), the PI compensator parameters are designed as follows:

$$
\begin{aligned}
& K_{p}=\frac{2 \zeta \omega_{n} u_{r}}{m h} \\
& K_{i}=\frac{\omega_{n}^{2} u_{r}}{m h}
\end{aligned}
$$

Here, $\omega_{\mathrm{n}}$ and $\zeta$ are design parameters which should be decided by the designer. Generally speaking, a higher $\omega$ and a larger $\zeta$ are preferable in the design of PI compensator parameters. However digital implementation of the NCTF controller limits the design parameters to maintain the closed-loop stability. Detailed discussion on the theoretical background of the NCTF control system can be seen in $[12,15]$.

Due to the fact that the NCT and the compensator are constructed from a simple openloop experiment of the plant, the exact model including the friction characteristic and the identification task of the plant parameters are not required to design the NCTF controller. Therefore, the controller design is simple and easy to implement in practical situation.

\section{Compensator improvement}

Since the NCTF controller uses PI compensator to force plant motion so that it follows the NCT, the integrator windup up may occur in connection with large position reference. As discussed in [15], in the case of no parameter variations, there is no significant integrator windup due to the effect of the saturation. The effect of the saturation is successfully compensated for using NCTF controller. However, the integrator windup becomes a problem when the parameters vary [15]. 
To overcome the problem of integrator windup, the PI compensator is improved by adopting an anti-windup scheme. Hence an anti-windup PI compensator is proposed to be used instead of a pure PI compensator. Here, a simple and classical tracking anti-windup is used [16]. The structure of the anti-windup PI compensator is illustrated in Fig. 4(a), where $\mathrm{K}_{\mathrm{T}}$ is called the tracking gain. Based on Fig. 4(a), once PI compensator output U(s) exceeds the actuator limits, a feedback signal is generated from the difference of the saturated and the unsaturated signals. This signal is used to reduce the integrator input. Mathematically, the output of the anti-windup PI compensator is

$$
U(s)=\left[K_{p}+\frac{K_{i}}{s}\right] U_{p}(s)-\frac{K_{T}}{s}\left[U(s)-U_{s}(s)\right]
$$

where $K_{p}$ and $K_{i}$ are proportional and integral gains obtained from section II.

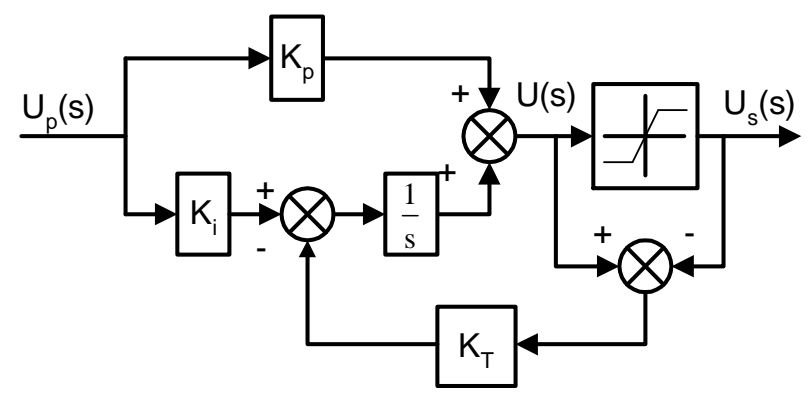

(a) Standard structure of anti-windup PI compensator

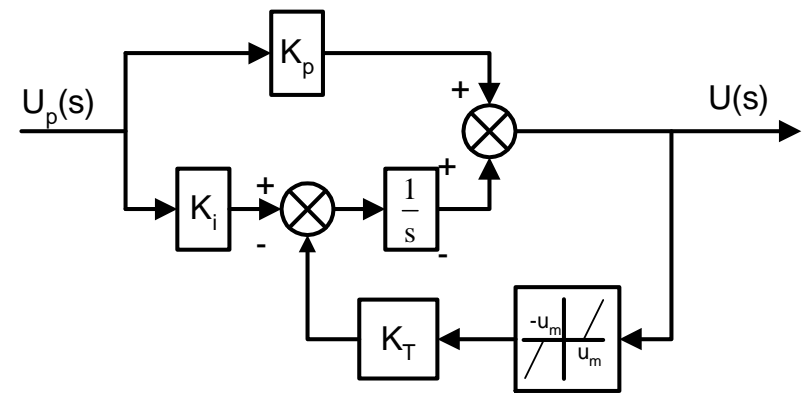

(b) Alternative structure of anti-windup PI compensator

Fig. 4: Proposed anti-windup PI compensator.

The anti-windup PI compensator, shown in Fig. 4(a), may be replaced by the structure shown in Fig. 4(b) where the unsaturated signal is used as input to the dead zone so that the feedback signal is generated. The dead zone range from $-u_{m}$ to $u_{m}$ represents the linear range of the actuator. Furthermore, the dead zone gain b relates with integral gain $K_{i}$ and tracking gain $\mathrm{K}_{\mathrm{T}}$ as follows: 


$$
b=\frac{K_{T}}{K_{i}}
$$

A rule of thumb for the setting of $K_{T}$ is often $K_{T}=K_{i}$ which corresponds to $b=1$, but a higher values may give a further improvement in performance [16].

\section{Results}

\subsection{System description}

The NCTF controller with anti-windup PI compensator is examined using a dynamic model of the experimental rotary positioning system as shown in Fig. 5. The positioning system consists of an AC servomotor, a driver and an inertia mass (spindle). For examining the positioning performance, the detailed model of Fig. 6 is used. Its parameters are shown in Table I. The positioning performance is examined under two conditions namely Normal Plant and Increased Inertia Plant. Normal Plant has the nominal plant parameters described in Table 1, while Increased Inertia Plant has about 10 times spindle inertia than that of Normal Plant.

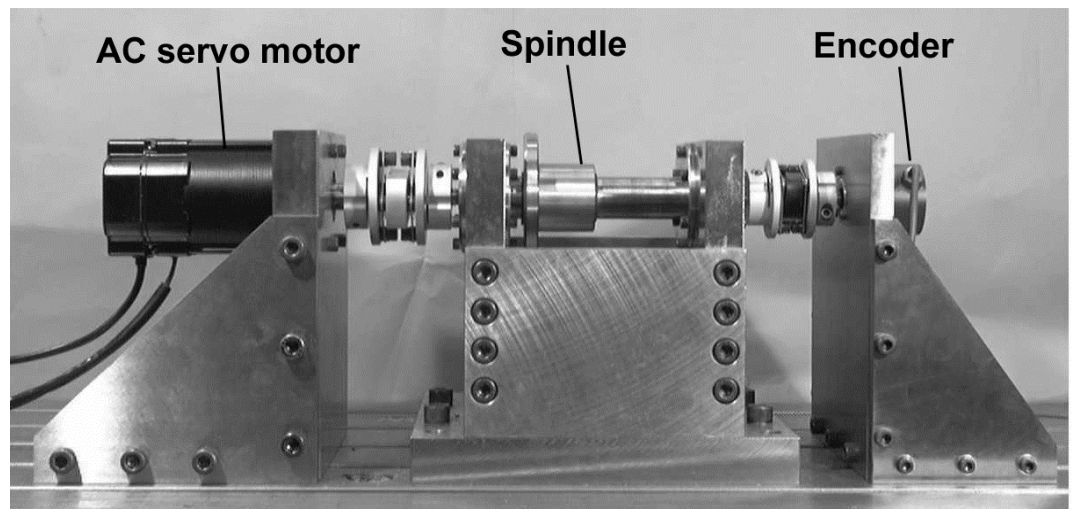

Fig. 5: Experimental rotary positioning system.

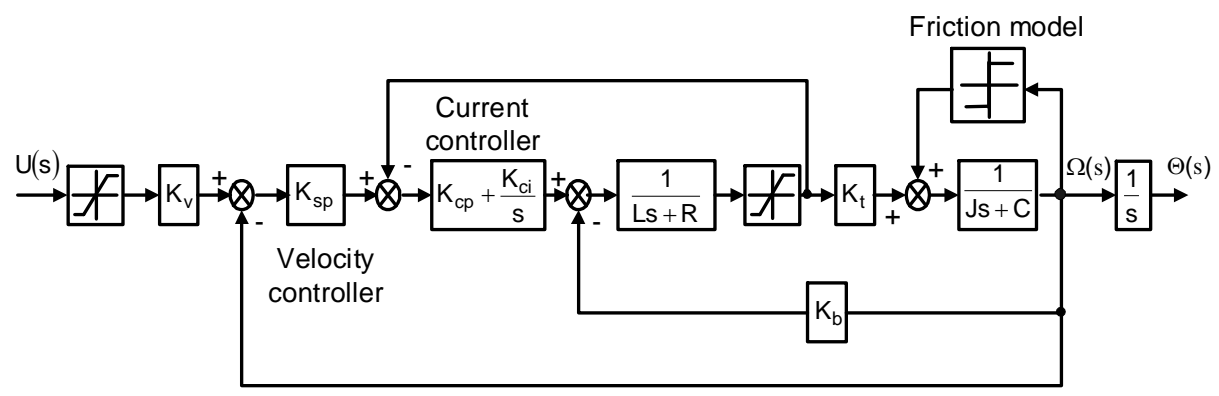

Fig. 6: Detailed model of rotary positioning system. 
Table I: Parameters of the Plant.

\begin{tabular}{ll}
\hline Parameter & Value \\
\hline Inertia load, $J$ & $1.17 \times 10^{-3} \mathrm{kgm}^{2}$ \\
Motor resistance, $R$ & $1.2 \Omega$ \\
Motor inductance, $L$ & $8.7 \mathrm{mH}$ \\
Motor torque constant, $K_{t}$ & $0.57 \mathrm{Nm} / \mathrm{A}$ \\
Back-Emf constant, $K_{b}$ & $0.57 \mathrm{Vs} / \mathrm{rad}$ \\
Viscous friction, $C$ & $1.67 \times 10^{-3} \mathrm{Nms} / \mathrm{rad}$ \\
Frictional torque, $\tau_{f}$ & $0.215 \mathrm{Nm}$ \\
Proportional current gain, $K_{c p}$ & $26.2 \mathrm{~V} / \mathrm{A}$ \\
Integral current gain, $K_{c i}$ & $3.62 \times 10^{3} \mathrm{~V} / \mathrm{As}$ \\
Proportional velocity gain, $K_{s p}$ & $8.60 \times 10^{-2} \mathrm{As} / \mathrm{rad}$ \\
Input voltage range, $u_{r}$ & $\pm 6 \mathrm{Volt}$ \\
\hline
\end{tabular}

\subsection{Controllers Design}

First, the NCTF controller is designed based on the Normal Plant. Figure 7 shows the $\mathrm{NCT}$ as a result of a simulated experiment. According to Fig. 7, the inclination $\mathrm{m}$ and maximum error rate of $\mathrm{h}$ of the NCT are $67.4 \mathrm{rad}$ and $240 \mathrm{rad} / \mathrm{s}$ respectively. The compensator parameters are designed by using $\mathrm{h}$ and $\mathrm{m}$ of the NCT. For designing the PI compensator, design parameters $\zeta$ and $\omega_{\mathrm{n}}$ are chosen as 13 and $29 \mathrm{rad} / \mathrm{s}$ respectively [15]. Table II shows the value of the compensator parameters calculated with (2).

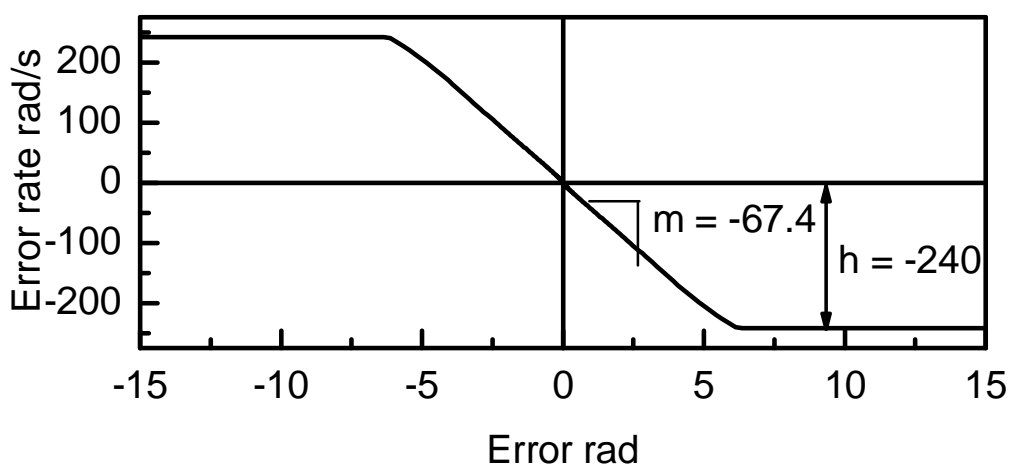

Fig. 7: Nominal characteristic trajectory (NCT).

The performance of the positioning system controlled by NCTF controller is compared with that controlled by PID controller. The PID controller is designed so that it has a similar bandwidth with the NCTF control system [15]. In this paper, the PID controller 
tuned with Ziegler-Nichols rule is not discussed again since it gives a bad robustness to parameter variations [15]. The PID controller parameters are also shown in Table II.

\subsection{The effect of the tracking gain $\mathrm{K}_{\mathrm{T}}$ on positioning performance}

The crucial problem for anti-windup PI compensator is the value of the tracking gain $\mathrm{K}_{\mathrm{T}}$. Although it is stated that a rule of thumb for the setting of $K_{T}$ is often $K_{T}=K_{i}$ which corresponds to $b=1$, but a higher value may give a further improvement in performance [16]. Therefore, in order to find the appropriate value of $K_{T}$, a simulation study has been carried out for different values of b which represent the ratio between tracking gain $K_{T}$ and integral gain $\mathrm{K}_{\mathrm{i}}$. Simulations have been done based on Increased Inertia Plant and large step input references (i.e. $5 \mathrm{rad}$ and $10 \mathrm{rad}$ step inputs) so that a saturation can occur. Moreover, the positioning performance is evaluated based upon maximum overshoot and $2 \%$ settling time.

Figure 8 shows the effect of the tracking gain $K_{T}$ on the positioning performance. Figure 8(a) clearly shows that the overshoot can be reduced by using anti-windup PI compensator. In addition, a larger gain tracking results in a smaller overshoot. As shown in Fig. 8(b), however, the settling time may become longer when a larger tracking gain $\mathrm{K}_{\mathrm{T}}$ is used. Therefore, the tracking gain should be decided based on the compromise between the overshoot and settling time. By considering a small enough overshoot and a shortest settling time, the gain ratio $b=12$ is selected to set the tracking gain $\mathrm{K}_{\mathrm{T}}$.

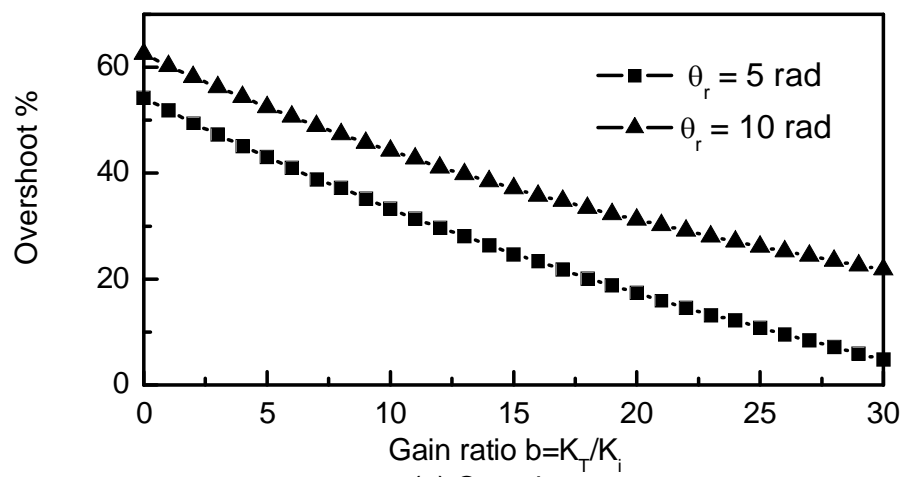

(a) Overshoot

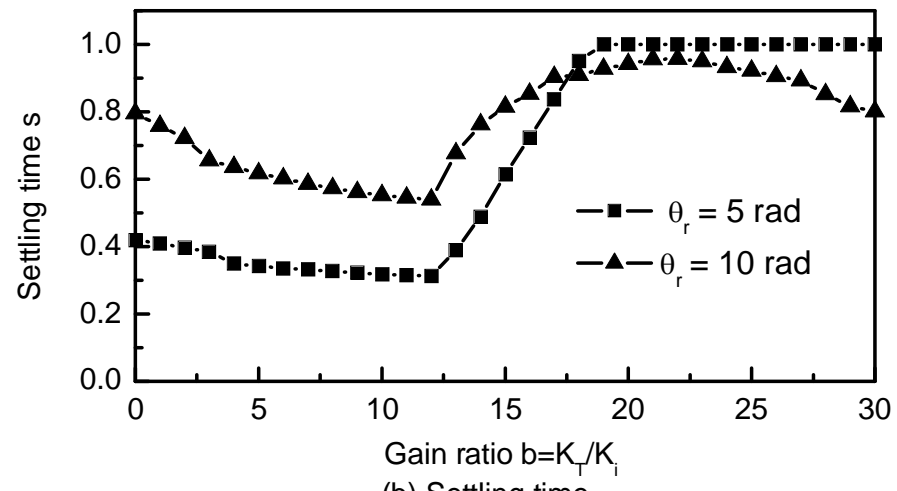

(b) Settling time

Fig. 8: Effect of tracking gain on positioning performance. 


\subsection{Comparison with PID controller}

In this section, the performance of the positioning system controlled by the NCTF (NCTF-2) controller with anti-windup PI compensator is compared with that of the normal NCTF (NCTF-1) and PID controllers. Figure 9 shows the step responses to 0.5 rad step input when the controllers are used to control Normal Plant. The positioning performance is summarized in Table III. As shown in Fig. 9(b), the 0.5-rad step input does not cause the saturation of the control signal. Here, it is clear that all controllers produce a similar response due to a similar bandwidth. Hence, in terms of overshoot and settling time, all the controllers produce similar performance.

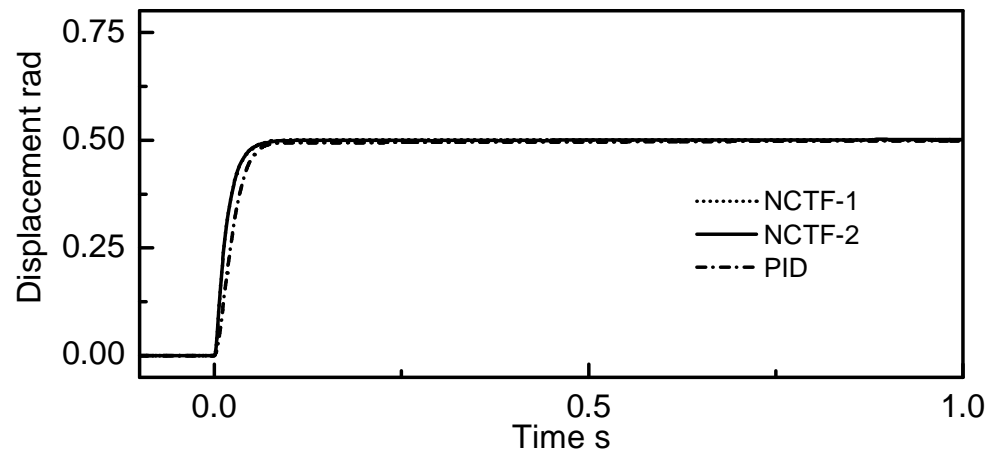

(a) Step responses to a 0.5-rad step input

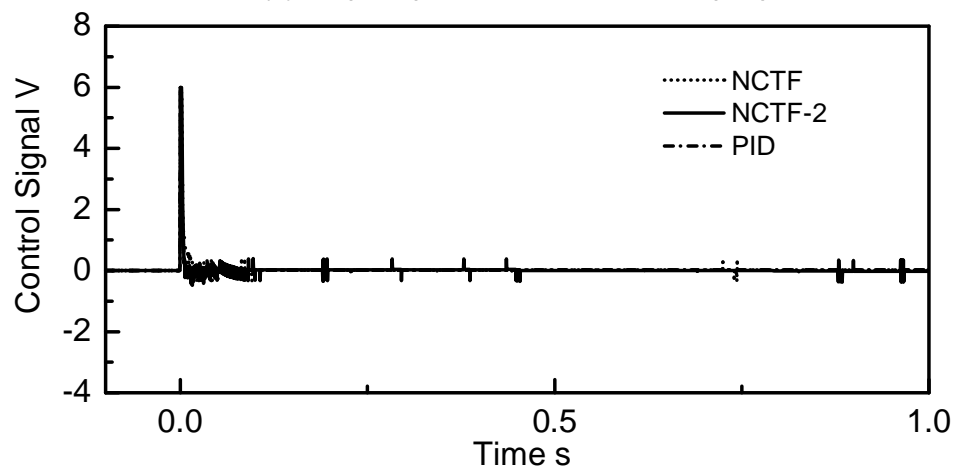

(b) Control Signal

Fig. 9: Comparison of response to a 0.5-rad step input, Normal Plant.

Moreover, in order to evaluate the robustness of the control systems to inertia variation, all the controllers are implemented on an Increased Inertia Plant. Figure 10 shows the step responses to a 0.5 -rad step input when all the controllers are implemented for controlling Increased Inertia Plant. Table III shows the positioning performance resulting from all the controllers. Figure 10 and Table III show that both NCTF controllers are more robust to inertia variation than the PID controller. Both NCTF controllers give similar results since there is no significant saturation of the actuator as shown Fig. 10(b). The result confirms that the use of anti-windup PI compensator does not affect the positioning performance when there is no saturation of the actuator. 


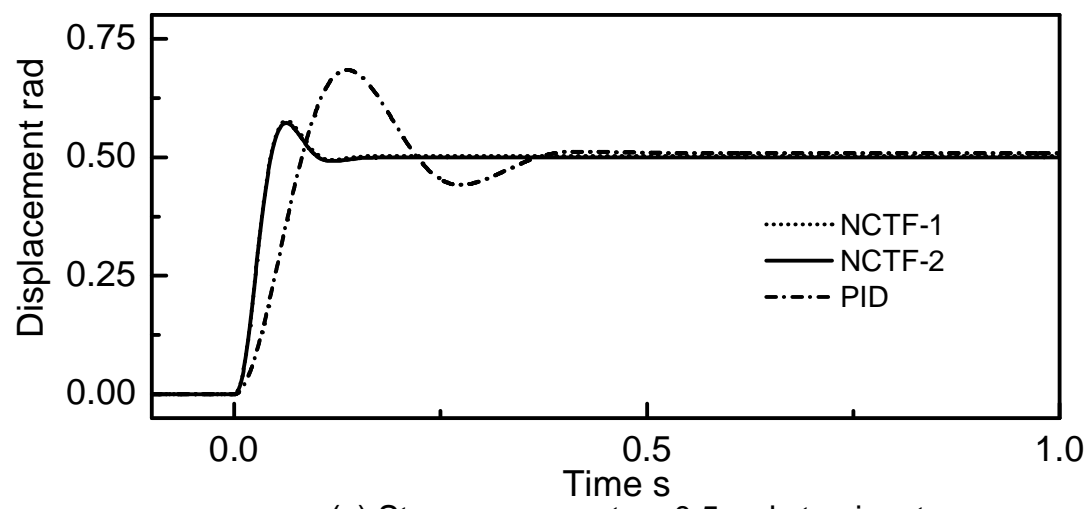

(a) Step responses to a 0.5 -rad step input

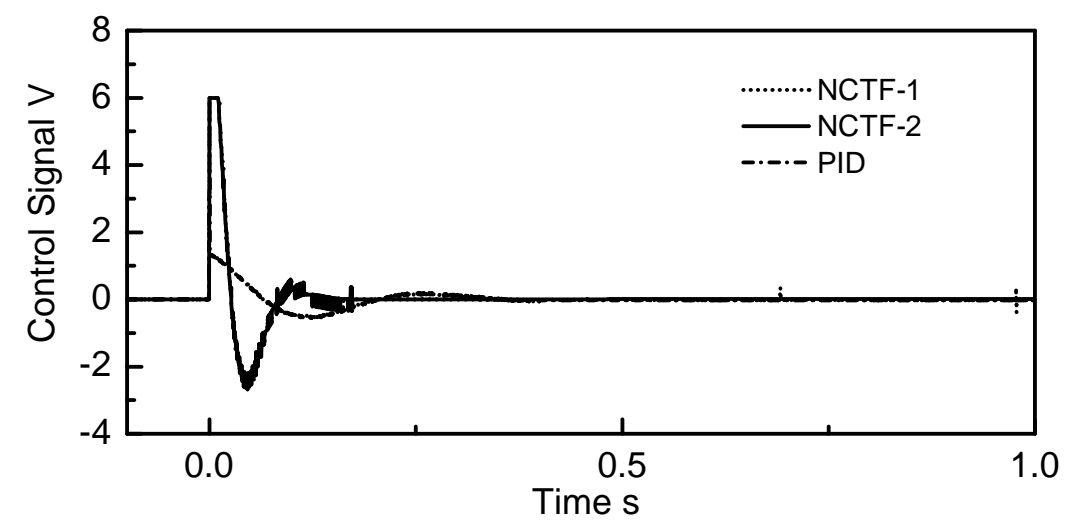

(b) Control Signal

Fig. 10 Comparison of response to a 0.5-rad step input, Increased Inertia Plant.

Next, simulation is done for a larger step input so that the actuator is saturated. Figure 11 shows the step responses to a 5 rad step input when all the controllers are implemented for controlling Increased Inertia Plant. Table IV shows the positioning performance resulting from all the controllers. The saturation of the actuator occurs as shown in Fig. 11(b). The saturation of the actuator causes an integrator windup when the positioning system is controlled by both NCTF-1 and PID controller. However, Fig. 11(a) and Table IV show that the positioning performance of the positioning system with NCTF-1 is worse than that of PID controller. Hence the NCTF-1 becomes less robust to inertia variation when the saturation occurs in comparison with that with PID controller. On the other hand, NCTF-2 which uses anti-windup PI compensator can successfully compensate the effect of integrator windup due to actuator saturation. As the results show, the improved NCTF controller (NCTF-2) gives a smaller overshoot and a shorter settling time than the other controllers. Hence it can be concluded that the improved NCTF controller which uses antiwindup PI compensator can maintain the robustness to parameter variation even if the actuator of the plant saturates. 


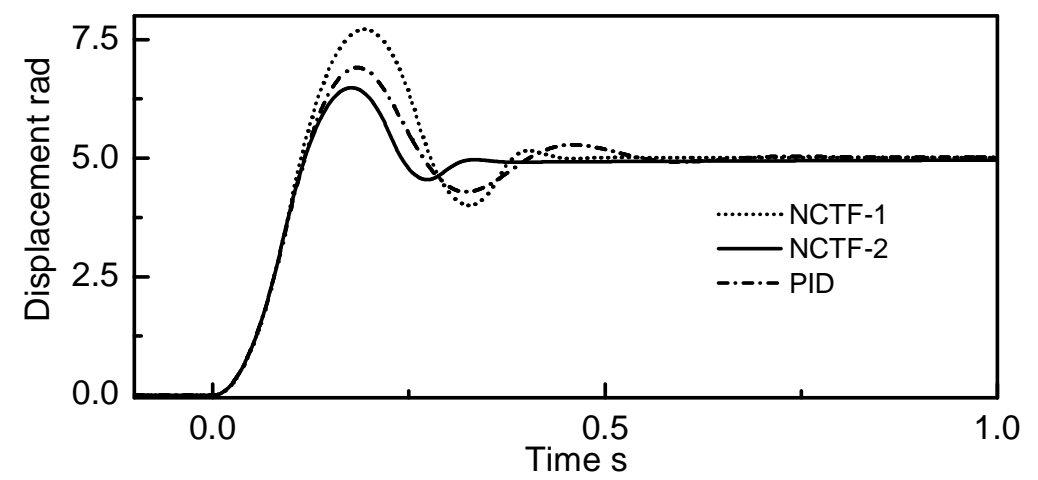

(a) Step responses to a 0.5 -rad step input

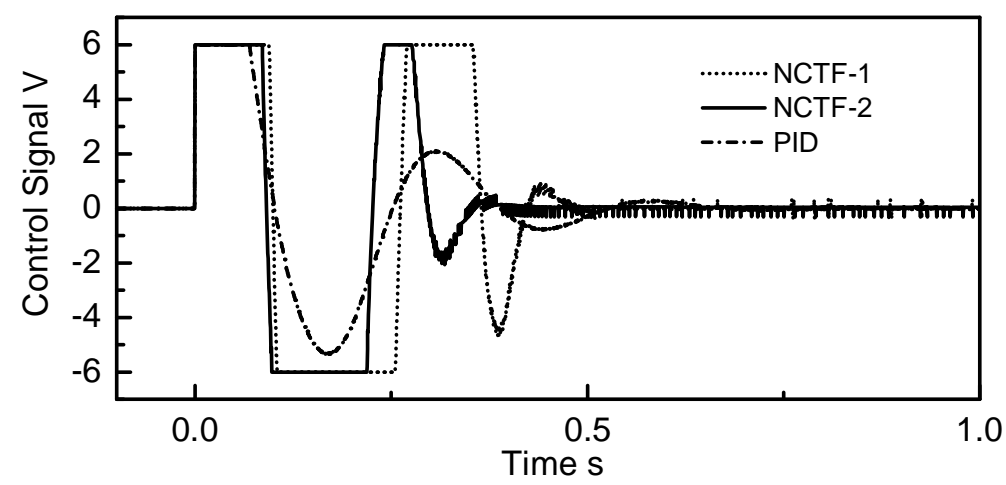

(b) Control Signal

Fig. 11: Comparison of response to a 5-rad step input, Increased Inertia Plant.

Furthermore, another simulation is done to compare the improved NCTF controller with anti-windup PID controller. For this purpose, an anti-windup scheme described in section III which is similar to that of NCTF controller is used. Two gain ratios for $b$ are used. The first anti-windup PID controller (PID-1) uses a gain ratio $b$ of 1 , in which the gain tracking is equal to integral gain of PID controller. While, the second anti-windup PID controller (PID-2) uses a gain ratio of 12, which is the same as the improve NCTF controller. Figure 12 shows the step responses to a 5-rad step input when all of the controllers are implemented for controlling Increased Inertia Plant. Table IV shows the positioning performance resulting from all of the controllers. Figure 12 and Table IV show that although the use of anti-windup PID controllers improves the robustness to inertia variation as compared to the normal PID controller, they are still less robust to inertia variation when compared to the improved NCTF controller. Hence, it can be concluded that the improved NCTF controller is much more robust than anti-windup PID controllers.

\section{Conclusions}

This paper has documented the improvement of the NCTF controller for overcoming the effect of windup due to actuator saturation. An anti-windup PI compensator is used as compensator of the NCTF controller instead of a conventional PI compensator. Through simulation, using rotary positioning system, the effectiveness of the NCTF controller with 
anti-windup PI compensator is evaluated. The results confirm that the use of anti-windup PI compensator is effective in overcoming the problem that arises from the integrator windup. Moreover, the obtained results also show that the NCTF control system with antiwindup PI compensator is much more robust to inertia variation than that of conventional PI compensator and the PID controllers.

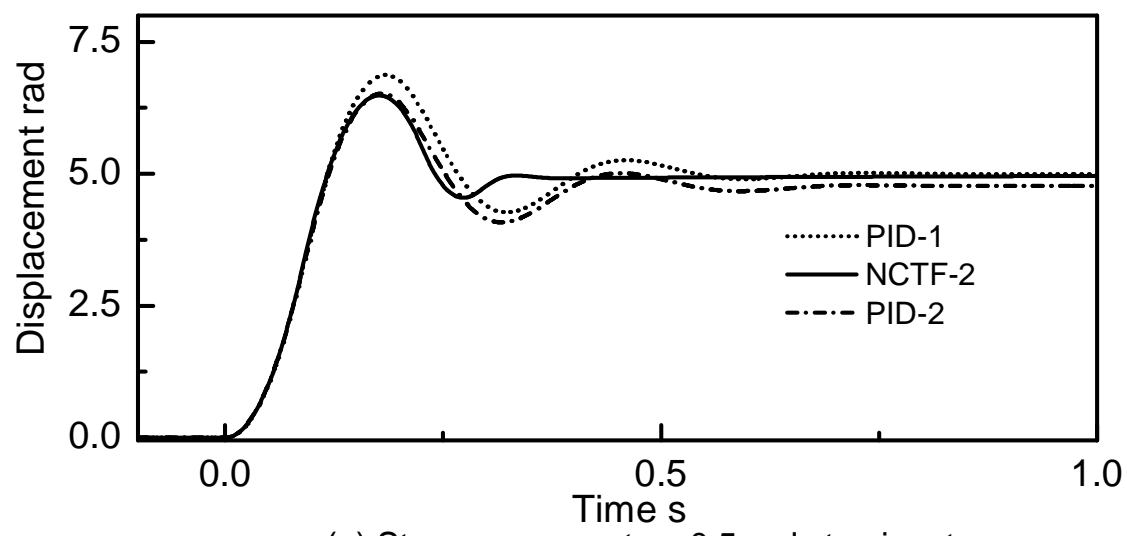

(a) Step responses to a 0.5-rad step input

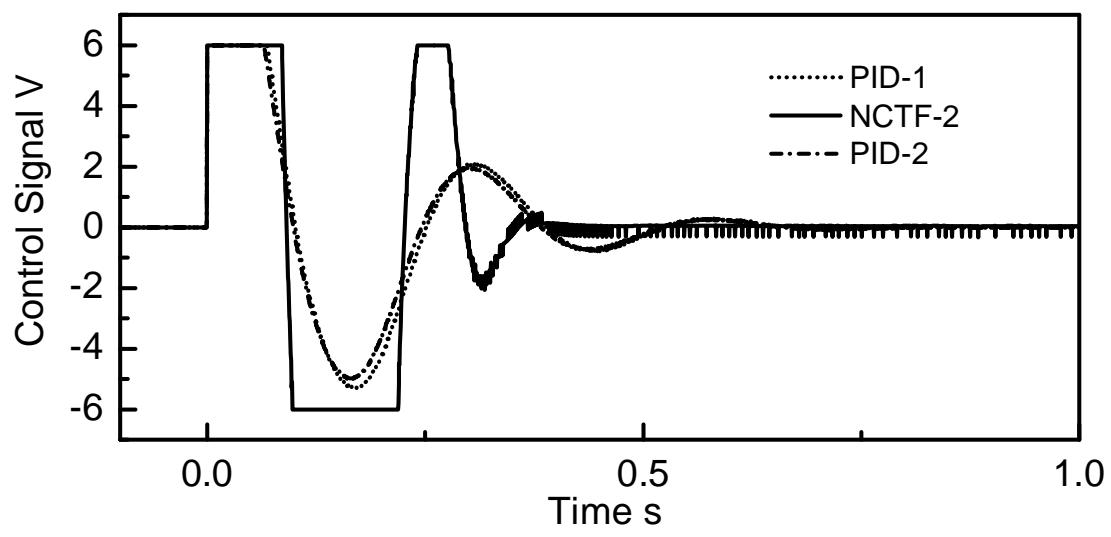

(b) Control Signal

Fig. 12: Comparison of response to a 5-rad step input, Increased Inertia Plant.

\section{References}

[1] K. Umeno, T. Kanoko and Y. Hori, "Robust Servosystem Design with Two Degree of Freedom and Its applications to Novel Motion Control of Robot Manipulators", IEEE Trans. on Industrial Electronics, 40 (5), pp. 473-485, 1993.

[2] S. Endo, H. Kobayashi, C.J. Kempf, S. Kobayashi, M. Tomizuka and Y. Hori, "Robust Digital Tracking Controller Design for High-speed Positioning systems", Control Engineering Practice, 4(4), pp. 527-536, 1996. 
[3] M. Tomizuka, "Robust Digital Motion Controllers for Mechanical Systems", Robotics and Autonomous Systems, 19, pp. 143-149, 1996.

[4] C. Kempf and S. Kobayashi, "Disturbance Observer and Feedforward Design for a Highspeed Direct-drive Positioning Table", IEEE Trans. on Control Systems Technology, 7(5), pp. 513-526, 1999.

[5] S. Wu and J. Fu, "Time-optimal Control of Servo Systems using PD Algorithms", JSME International Journal: Series C, 41(3), pp. 384-390, 1998.

[6] M.H. Park and C.Y. Won, "Time Optimal Control for Induction Motor Servo System”, IEEE Trans. on Power Electronics, 6(3), pp. 514-524, 1991.

[7] M.L. Workman, R.L. Kosut and G.F. Franklin, "Adaptive Proximate Time-optimal Servomechanisms: Continuous Time Case", Proceedings of the American Control Conference, Minneapolis, USA, pp. 589-594, 1987.

[8] C.J. Kempf, "Step and Settle Positioning Algorithm for Electro-mechanical System with Damping", Proceedings of the $4^{\text {th }}$ International Workshop on Advanced Motion Control, Tsukuba, Japan , pp. 47-52, 1996.

[9] S. Sankaranarayanan and F. Khorrami, "Adaptive Variable Structure Control and Applications to Friction Compensations", Proceedings of the $36^{\text {th }}$ IEEE Conference on Decision \& Control, San Diego, USA, pp. 4159-4164, 1997.

[10] Y. Fujimoto and A. Kawamura, "Robust Servo-system Based on Two-degree-of-freedom Control with Sliding Mode", IEEE Trans. on Industrial Electronics 42(3), pp. 272-280, 1995.

[11] Wahyudi, "New Practical Control of PTP Positioning Systems", Ph.D Thesis, Tokyo Institute of Technology, 2002.

[12] Wahyudi, K. Sato and A. Shimokohbe, "Robustness Evaluation of New Practical Control Method for PTP Positioning Systems", Proceeding of 2001 IEEE/ASME International Conference on Advanced Intelligent Mechatronics, pp. 843-848, 2001.

[13] Wahyudi, K. Sato and A. Shimokohbe, "New Practical Control Method for PTP Positioning Systems: Robustness Evaluation", Proceeding of $10^{\text {th }}$ International Conference on Precision Engineering, pp. 774-778, 2001.

[14] Wahyudi, "Robustness Evaluation of Two Control Methods for Friction Compensation of PTP Positioning Systems", Proceeding of 2003 IEEE Conference on Control Applications, pp. 1454-1458, 2003.

[15] Wahyudi, K. Sato and A. Shimokohbe, "Characteristics of Practical Control for Point-topoint (PTP) Positioning Systems: -Effect of design parameters and actuator saturation on positioning performance", Precision Engineering, Vol. 27, pp. 157-169, 2003.

[16] C. Bohn and D.P. Atherton, "A SIMULINK Package for Comparative Studies of PID Antiwindup Strategies", Proceeding of IEEE/IFAC Joint Symposium on Computer-Aided Control System Design, pp. 447-452, 1994.

\section{BIOGRAPHIES}

Wahyudi was born in Indonesia in July 1970. He received his B.Eng and MSc degrees in mechanical engineering from Institute of technology Bandung, Indonesia in 1994 and 1997 , respectively and his Ph.D. in precision machinery systems from Tokyo Institute of Technology, Tokyo, Japan, in 2002. He is currently an assistant professor at the Mechatronics Engineering Department, International Islamic University Malaysia. His 
research fields are motion control, Mechatronics and intelligent systems. Dr. Wahyudi is a member of IEEE.

Tarig Faisal was born in Khartoum, Sudan 1978. He received his B.Sc. degree in Electronic Engineering from the University of Jazeera in 2001. He joined Nor Al Eyon company as a control engineer in 2001. Currently, he is a master student at Mechatronics Engineering Department, International Islamic University Malaysia. His primary research interests are design, analysis and performance evaluation of intelligent as well as industrial control systems.

Abdulgani Albagul received his B.Sc. degree in electronic engineering from the higher institute of electronics in Baniwalid, Libya in 1989, MSc, in control engineering from university of Bradford in 1993 and Ph.D. in engineering from University of Newcastle upon Tyne in 2001. He is currently an assistant professor at the Department of Mechatronics Engineering, Faculty of Engineering at the International Islamic University Malaysia. His research interests are control systems, system dynamics and modeling, smart sensor and instrumentation, robotics and automation. He is a member of IEEE and he has several publications. 